\title{
BMJ Open Quality Managing high frequency users of an electronic consultation system in primary care: a quality improvement project
}

Katherine Leung (D) , Saffan Qureshi

To cite: Leung K, Qureshi S. Managing high frequency users of an electronic consultation system in primary care: a quality improvement project. BMJ Open Quality 2021;10:e001310. doi:10.1136/ bmjoq-2020-001310

Received 28 December 2020 Accepted 29 May 2021

\section{Check for updates}

(C) Author(s) (or their employer(s)) 2021. Re-use permitted under CC BY-NC. No commercial re-use. See rights and permissions. Published by BMJ.

Whitechapel Health Centre, London, UK

Correspondence to Dr Katherine Leung; katherine.leung@nhs.net

\section{ABSTRACT}

The COVID-19 pandemic prompted a rapid change in primary care provision. There was a significant shift from face-to-face appointments to remote methods such as electronic consultation (e-consultation). Patients from a primary care provider in London were actively encouraged to use an online consultation platform called 'Dr iQ'. A group of high frequency users of Dr iQ emerged and clinicians were concerned their health needs were not being met through the platform. High frequency attendance in a traditional general practice setting is associated with increased time and healthcare costs. This project evaluated the number of high frequency users (identified as 10 or more consultations a month) of $\mathrm{Dr}$ iQ in one busy inner city practice over a 5-month period. We aimed to decrease the subsequent monthly usage frequency of all $\mathrm{Dr} i \mathrm{Q}$ high frequency users from 10 or more consultations to less than 10 consultations. Our interventions included a semi-structured telephone interview, discussion among the multidisciplinary team, and regular scheduled telephone or face-to-face appointments. Following two Plan-Do-Study-Act cycles, all 12 high frequency users showed a decrease in the number of consultations submitted to $\mathrm{Dr}$ iQ to less than 10 consultations a month.

This project proposes a method of case managing high frequency users of e-consultation. The majority of high frequency users had unmet health needs and felt a lack of continuity of care on Dr iQ. They often had complex physical and mental health problems. As remote consulting technology continues to develop, more research is required to understand the epidemiology and aetiology of e-consultation high frequency use in order to improve patient outcomes.

\section{PROBLEM}

The COVID-19 pandemic necessitated a swift and drastic transformation in primary care provision. Consultations shifted rapidly from face-to-face appointments to virtual methods, such as telephone and electronic consultation (e-consultation), to prevent the spread of the virus and protect patients and staff members. From April 2020, patients of 38 practices from a large regional primary care provider in London were actively encouraged to download and use an online consultation system called 'Dr iQ'. '

There emerged a group of patients submitting consultations on $\mathrm{Dr}$ iQ to a high frequency with a subgroup of patients submitting consultations in an inappropriate manner. In the traditional general practice setting, it has been reported the top $10 \%$ of highest attending patients have a mean of 28 consultations a year, even when stratified for comorbidity. ${ }^{2}$ In July $2020,31 \%(10 / 32)$ of practices reported at least 1 patient submitting 15 consultations or more a month on $\mathrm{Dr}$ iQ; and even though some consultations may be attributed to administrative tasks such as requesting sick notes or repeat medication, this is still a significantly higher rate than 28 consultations a year.

There were concerns from the clinical team about whether these patients' health needs were being met and whether they were suited to the Dr iQ platform. This project focused on one busy inner city practice as a case study to further explore high frequency use of Dr iQ. At the time of writing, the practice had a list size of 13528 patients, with $28 \%$ of adult patients at the practice registered with the $\mathrm{Dr}$ $i Q$. The median age range at the practice is 25-29 years old. ${ }^{3}$

We proposed to evaluate the number of high frequency users of Dr iQ monthly over a 5 -month period. Our aim was to decrease the subsequent monthly usage frequency of all Dr iQ high frequency users from 10 or more consultations to less than 10 consultations.

\section{BACKGROUND}

In recent years, there has been a significant drive towards digital methods of patient access to primary care. The National Health Service long-term plan, published in January 2019, set out an aim for a 'digital-first primary care' service and committed that every patient has a right to access their general practitioner (GP) 
through digital methods by $2023 / 2024 .{ }^{4}$ The benefits of e-consultations have been attributed to better patient convenience and accessibility as well as improved work efficiency to meet the increasing demand on primary care. $^{56}$

In 2016, a survey conducted by the Royal College of General Practitioners (RCGP) found that despite policy pressure, GPs were generally reluctant to move away from face-to-face consultation methods, and the majority of GPs had no future plans to implement e-mail or video consultations. ${ }^{7}$ Prior to COVID-19, $82 \%$ of consultations were performed face-to-face and $15 \%$ were through remote methods. ${ }^{8}$

An RCGP survey conducted in July 2020 demonstrated the vast effects of the pandemic on primary care: GPs were now carrying out $87 \%$ of consultations remotely and only $13 \%$ of consultations face-to-face. ${ }^{9}$ The survey further showed that the majority $(>67 \%)$ of GPs found that remote consultation methods (telephone, e-consultations, video) increased efficiency.

\section{Dria}

Dr iQ developed by AT Medics, is a website and mobile application providing patients a platform to conduct online consultations. ${ }^{1}$ After registering with the platform, patients can conduct audio, video or e-consultations, with the ability to attach clinically relevant information such as pictures, as well as administrative tasks such as ordering repeat medications and requesting sick notes. Algorithms with screening questions are utilised for e-consultations relating to common patient presentations. Online consultations are reviewed by a group of clinicians including clinical pharmacists, nurse practitioners, and GPs. E-consultations can be either closed remotely through messaging on the platform, or, if necessary, followed up with a face-to-face or telephone appointment.

As more patients registered for Dr iQ, use of the platform steeply increased to an average of 1500 consultations per month per practice, with one practice processing 3600 consultations in September 2020. There were many patient-reported and clinician-reported benefits to the platform. From in-app patient feedback, patients report in $45 \%$ of cases Dr iQ prevented a face-to-face appointment and in $39 \%$ of cases prevented an attendance to urgent care. With the platform available 24 hours a day, 7 days a week, patients favoured the increased accessibility and decreased waiting times compared with the traditional general practice model.

\section{High frequency users}

High frequency attendance (HFA) in primary care is well documented with publications on the subject from the $1960 \mathrm{~s} .{ }^{10}$ It has been particularly well researched in Denmark where, similar to the UK, a publicly funded healthcare system means there is a near to no financial barrier to access primary care. ${ }^{11}$ HFA has been associated with pre-existing medical and psychiatric conditions, female gender, low levels of education, unemployment, and living alone. ${ }^{11-14}$ HFA is associated with increased time and healthcare costs. ${ }^{15}$ It has been estimated that $80 \%$ of a GPs time is spent on $20 \%$ of patients and one in every seven consultations with the top $3 \%$ of frequent attenders. ${ }^{16}$ Though most HFA is transient, a proportion continue to be persistent frequent attenders. ${ }^{17}$

A number of randomised controlled trials have studied the effects of interventions on HFA, including cognitive behavioural therapy, group interventions and educational programmes, and a systematic review suggested in-depth analysis of reasons for HFA may reduce consultation frequency. ${ }^{18}$ However, no research relating to high frequency users of online consultations has been published at the time of writing. This may become an important focus for research in the future if digital methods of consulting are here to stay.

\section{MEASUREMENT}

There are many different definitions of frequent attendance, including the top percentage of consultation frequency (most frequently top 10\%) and a cut-off number ranging from 5 to 24 consultations a year. ${ }^{2} 1418$ Looking at our baseline data, 1727 users submitted at least one consultation in July 2020. We identified 46 users who submitted 5 or more consultations on DriQ with 6 of them submitting 10 consultations or more. As we wanted to focus our project on a sample size that was neither too large to introduce difficulties in intervention, nor too small to affect the quality of data, we decided on defining high frequency users as users who submit 10 or more consultations per month on Dr iQ. The consultations could be a mix of medical queries, medication requests, sick note requests, and general queries (e.g., chasing up referrals or requesting a blood test). They excluded audio and video consultations.

High frequency users were identified monthly between July and November 2020 and their notes reviewed to see if usage of the platform was appropriate or inappropriate. Appropriateness was assessed on a case-by-case basis, but most commonly, inappropriate use of Dr iQ was defined as such if users submitted numerous consultations about the same clinical problem within a short period of time. Inappropriate use may also be submitting a high frequency of consultations with minimal or irrelevant information. On the contrary, an example of appropriate high frequency use would be submitting a variety of queries and/or consultations for different family members.

\section{DESIGN}

The quality improvement team included a general practice specialty registrar and GP who was clinical lead of the practice. As the team responding to Dr iQ consultations consisted of a number of allied healthcare professionals, we endeavoured to keep the multidisciplinary team updated at regular intervals throughout the project. Each Plan-Do-Study-Act (PDSA) cycle was discussed and 
agreed with by key stakeholders including GPs, pharmacists, and nurse practitioners.

In order to inform our PDSA cycles, we realised the importance of gathering feedback from users to explore what factors were driving high frequency use. This was also informed by systematic review evidence suggesting that in-depth review of the reasons for frequent attendance may decrease consultation frequency. ${ }^{18}$ This part of the project involved a more qualitative approach in the form of a semi-structured telephone interview. It was anticipated that certain high frequency users may react negatively to being 'pointed out' about their use. As such, we emphasised to patients the purpose of the interview was not to reprimand them but rather to allow for an open discussion about their experience with Dr $\mathrm{iQ}$ and how we could improve our service to meet their health needs.

The focus of this project was not to deter high frequency users of Dr iQ from accessing healthcare but rather to assess whether their healthcare needs are being met through an online consultation platform, and if not, finding alternative methods to better suit their needs and ultimately improve patient care.

From reviewing the electronic health records of six high frequency users in July 2020, we noted that the majority of the patients were previously seen in the practice for complex physical and mental health problems, for example complex regional pain syndrome, medically unexplained symptoms and health anxiety. We suspected that for these individuals the Dr iQ platform would not be best suited for them as it can miss the nuances and interaction of a traditional GP consultation, potentially leading to unmet health needs. Further, there tended to be a lack of continuity of care with different healthcare professionals responding to each of their e-consultations. For these reasons, we planned to convert their consultation method from Dr iQ to regular face-to-face or telephone appointments with one named clinician.

We planned to make our intervention sustainable by appointing a member of the administrative team to run a monthly search to identify high frequency users and to bring them to the weekly multidisciplinary team for discussion.

\section{STRATEGY}

A graphical flow chart illustration of the PDSA cycles is provided in figure 1 .

\section{Plan-Do-Study-Act 1}

High frequency users deemed to be using Dr iQ inappropriately were telephoned in the first week of the following month they were identified and a semi-structured telephone interview took place, ranging from 5 to 10 minutes in length. Notes with verbatim quotes were made for all interviews.

They were first informed of the number of online consultations they had submitted the previous month, then their views of the consultation platform were assessed. Topics covered included whether the patient felt their health needs were being met by Dr iQ and their perceived advantages and disadvantages of the service. Finally, if appropriate, a suggestion to convert to regular telephone or face-to-face appointments with a single named clinician rather than using the online consultation platform was made.

This cycle led to a decrease in consultation frequency for all 12 high frequency users (deemed to be using Dr $\mathrm{iQ}$ inappropriately) in the month following intervention. In PDSA 1, 2 users over 5 months opted for conversion to regular appointments.

Several interesting insights arose from the telephone interviews relating to the reasons for high frequency usage and whether high frequency users' health needs were being met. A number of patients felt supported by the service and favoured the ease of access and speed of response from a clinician. One particular user reported they frequently forget to attend doctors' appointments and prefer to communicate their health queries through text which made Dr $\mathrm{iQ}$ an ideal platform. They felt having ease of access to healthcare professionals decreased their health anxiety and prevented them from 'Googling' their symptoms. Other users echoed a favour for the speed of response:

"When I message them today they will call me straight away"

DR-IQ-8

"I like how every time I send a request I get an answer straight away"

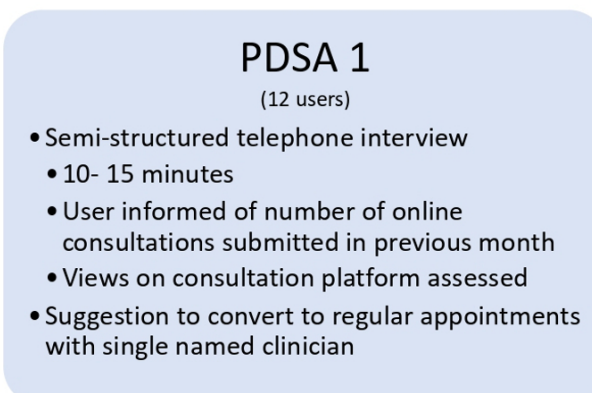

3/12 users continued to submit high levels of consultations or inappropriate consultations following PDSA 1

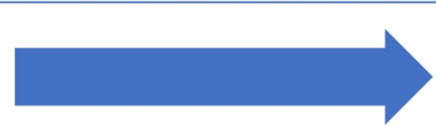

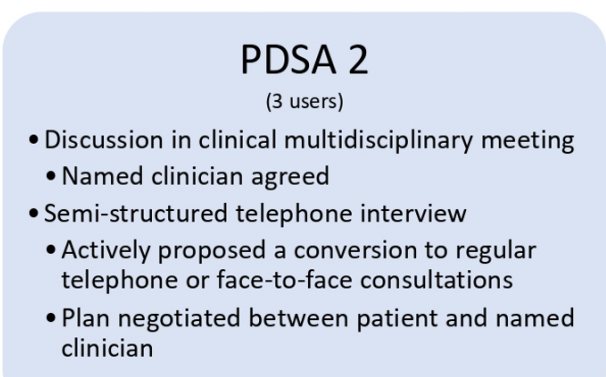

PSA 2

- Discussion in clinical multidisciplinary meeting

(amed clinician agreed

- Actively proposed a conversion to regular

- Plan negotiated between patient and name clinician

Figure 1 A graphical flow chart illustration of the Plan-Do-Study-Act (PDSA) cycles. 
DR-IQ-11

Interestingly and unexpectedly, high frequency users who felt their health needs were being met by Dr iQ decreased their usage after the telephone interview. It is difficult to attribute this decrease to the effect of our intervention or other factors, as users could well have been experiencing an acute health event leading to higher use which would have naturally decreased following the event. On the contrary, it may highlight the effect of human interaction and listening to users for their feedback. Informing users of their frequency of usage could have also led to behaviour change. One user in particular said the following after being told about their monthly usage:

"I had no idea! Has it really been that bad?... I'm trying to find something negative [about Dr $i Q] \ldots$ nothing comes to mind"

DR-IQ-11

For others, the main reason for high frequency usage appeared to be unmet expectations. For example, one user was diagnosed with hypertension after submitting their home blood pressure readings on Dr iQ and subsequently started on ramipril. They were struggling to understand the diagnosis and management plan through e-consultations which contributed to them submitting repetitive queries. The telephone interview provided an opportunity for an interactive, thorough explanation of hypertension with the patient.

"I have high blood pressure, I have been trying to get in touch with the doctor to explain what I need to do, what medication... I've had 2 blood tests in the space of 3-4 weeks and I have no idea what is going on"

DR-IQ-14

The majority of high frequency users with unmet health needs felt there was a lack of continuity of care on Dr iQ. These patients tended to have complex chronic medical conditions as well as underlying mental health issues such as anxiety, depression, post-traumatic stress disorder, and medically unexplained symptoms:

"When I see my GP they know my condition and my situation, sometimes I want to see a GP for specific things... My only fear is that someone else takes the consultation and they don't check I've got underlying problems"

DR-IQ-2

"Whoever takes the consultation is aware of the whole history rather than that specific problem... Dr iQ clinicians can miss out points from previous encounters"

\section{DR-IQ-6}

It was this group of patients that informed our second PDSA cycle, as despite the telephone interview and suggestion of converting to regular face-to-face or telephone appointments they continued to use Dr iQ to a high frequency.

\section{Plan-Do-Study-Act 2}

The consultation frequency of high frequency users continued to be monitored every month after PDSA 1. PDSA 2 focused on users who continued to submit high levels of consultations (10 or more consultations a month) or inappropriate consultations (even if less than 10 a month) following PDSA 1. 3 out of 12 users were identified as such.

As with PDSA 1, consultations of persistent high frequency users were reviewed for appropriateness. They were discussed in a clinical multidisciplinary meeting with GPs, nurse practitioners, clinical pharmacists, and practice managers present. Any clinical concerns about the patient were explored as well as suggested avenues for further support. They were given a named clinician to aid with continuity of care.

This group was contacted again for a semi-structured telephone interview. Topics covered were similar in nature to the first telephone interview, however, in this cycle, there was more emphasis on actively proposing a conversion to regular telephone or face-to-face consultations. A plan was negotiated between the patient and their named clinician for an agreed mode of regular follow-up. This was often fortnightly or monthly appointments until the patient felt their health needs were met. They would then be discharged but given the option to make further follow-up appointments with their named clinician on an 'as required' basis.

Further, persistent high frequency users were educated on more suitable methods to use Dr iQ going forward. Specifically, for chronic or complex symptoms they were advised to book a consultation with their named clinician, allowing for continuity of care and more in-depth exploration of their health needs. They were advised Dr iQ would be appropriate for administrative tasks such as medication requests and sick notes.

As one of the persistent high frequency users (DR-IQ13) was identified in the last month of the project, they received the PDSA 2 intervention however their subsequent consultation frequency was not included in the results.

\section{RESULTS}

The number of high frequency users identified between July to November 2020 are listed in table 1. Table 2 details

Table 1 Number of high frequency users between July and November 2020, as defined by $\geq 10$ consultations submitted to $\mathrm{Dr}$ iQ per month

\begin{tabular}{ll}
\hline Month & No of high frequency users \\
\hline July & 5 \\
August & 3 \\
September & 2 \\
October & 5 \\
November & 0 \\
\hline
\end{tabular}


Table 2 The number of consultations submitted to Dr iQ by high frequency users from July to November 2020

\begin{tabular}{|c|c|c|c|c|c|c|c|}
\hline User & July & August & September & October & November & Intervention & $\begin{array}{l}\text { No of subsequent } \\
\text { telephone/face-to-face } \\
\text { appointments }\end{array}$ \\
\hline DR-IQ-1 & $13^{*}$ & 5 & 0 & 1 & 2 & TC & \\
\hline DR-IQ-2 & $12^{*}$ & $8^{*}$ & 4 & 3 & 1 & $\begin{array}{l}\text { TC, MDT, named } \\
\text { clinician }\end{array}$ & $\begin{array}{l}\text { Three over } 6 \text { weeks, then } \\
\text { discharged }\end{array}$ \\
\hline DR-IQ-3 & $11^{*}$ & 2 & 2 & 1 & 0 & $\begin{array}{l}\text { TC, opted for regular } \\
\text { appointments }\end{array}$ & Three over 6 weeks, ongoing \\
\hline DR-IQ-4 & $10^{*}$ & 1 & 1 & 1 & 0 & $\begin{array}{l}\text { TC, opted for regular } \\
\text { appointments }\end{array}$ & $\begin{array}{l}\text { Two over } 2 \text { months, then } \\
\text { discharged }\end{array}$ \\
\hline DR-IQ-5 & $10^{*}$ & 2 & 0 & 0 & 1 & TC & \\
\hline DR-IQ-6 & & $12^{*}$ & $7^{*}$ & 1 & 1 & $\begin{array}{l}\text { TC, MDT, named } \\
\text { clinician }\end{array}$ & Nine over 3 months, ongoing \\
\hline DR-IQ-10 & & & 10 & 1 & 5 & None, appropriate use & \\
\hline DR-IQ-11 & & & & $12^{*}$ & 3 & TC & \\
\hline DR-IQ-12 & & & & 12 & 3 & None, appropriate use & \\
\hline DR-IQ-13 & & & & $11^{*}$ & $9^{*}$ & $\begin{array}{l}\text { TC, MDT, named } \\
\text { clinician }\end{array}$ & Ongoing \\
\hline DR-IQ-14 & & & & $10^{*}$ & 2 & TC & \\
\hline DR-IQ-15 & & & & $10^{*}$ & 1 & TC & \\
\hline
\end{tabular}

The intervention given to each user and the number of subsequent telephone or face-to-face appointments (if applicable) are also listed. An asterisk next to the number signifies inappropriate platform use.

MDT, multidisciplinary team discussion; TC, telephone call.

the number of high frequency users' consultation submissions over time, the intervention given to each user, and the number of subsequent regular appointments if applicable. Figure 2 provides a graphical illustration of the trend of high frequency users' Dr iQ consultation frequency over time.

\section{LESSONS AND LIMITATIONS}

Following two PDSA cycles, we achieved our aim of decreasing the number of consultations submitted to Dr iQ by high frequency users from 10 or more consultations to less than 10 consultations a month. This was achieved for all 12 high frequency users identified monthly over a 5-month period and deemed to be using the platform inappropriately. Further, following PDSA 2 there was a sustained decrease in consultation use for persistent high frequency users DR-IQ-2 and DR-IQ-6.

Our usage data suggests that new high frequency users of an e-consultation platform will arise every month, similar to the pattern of HFA over time in a traditional GP setting. ${ }^{19}$ This highlights the importance of regular, continuous monitoring and intervention of high frequency users. We, therefore, implemented a system change whereby we appointed a senior member of the administrative team to run a monthly search for patients submitting 10 or more online consultations and to bring this list to the first weekly clinical meeting of each month. The multidisciplinary team will then discuss the patients, review their e-consultations for appropriateness, and nominate a named clinician, whose responsibility will be to telephone the patient, assess their health needs and suggest a conversion from Dr iQ to regular appointments.

As the usage of Dr iQ grows over time, we anticipate that the proportion of high frequency users will similarly rise. This project proposes a number of interface-related changes which will be discussed with the platform developer team. It would be beneficial to create an algorithm which automatically identifies new high frequency users each month and highlights them to the clinical team, thereby saving administrative time and labour. Second, user education on appropriate use of Dr iQ could be integrated as part of the sign up process for the platform. This could be in the form of a short video or notice explaining the types of consultation the platform is most suited for, and describes examples of occasions when it would be more appropriate to contact the surgery for a telephone or face-to-face appointment instead.

There were a number of strengths of this project. There was a strong emphasis on identifying unmet health needs and improving patient outcomes throughout. This was done on an individual level through the telephone interview. The interview provided valuable insight into how 

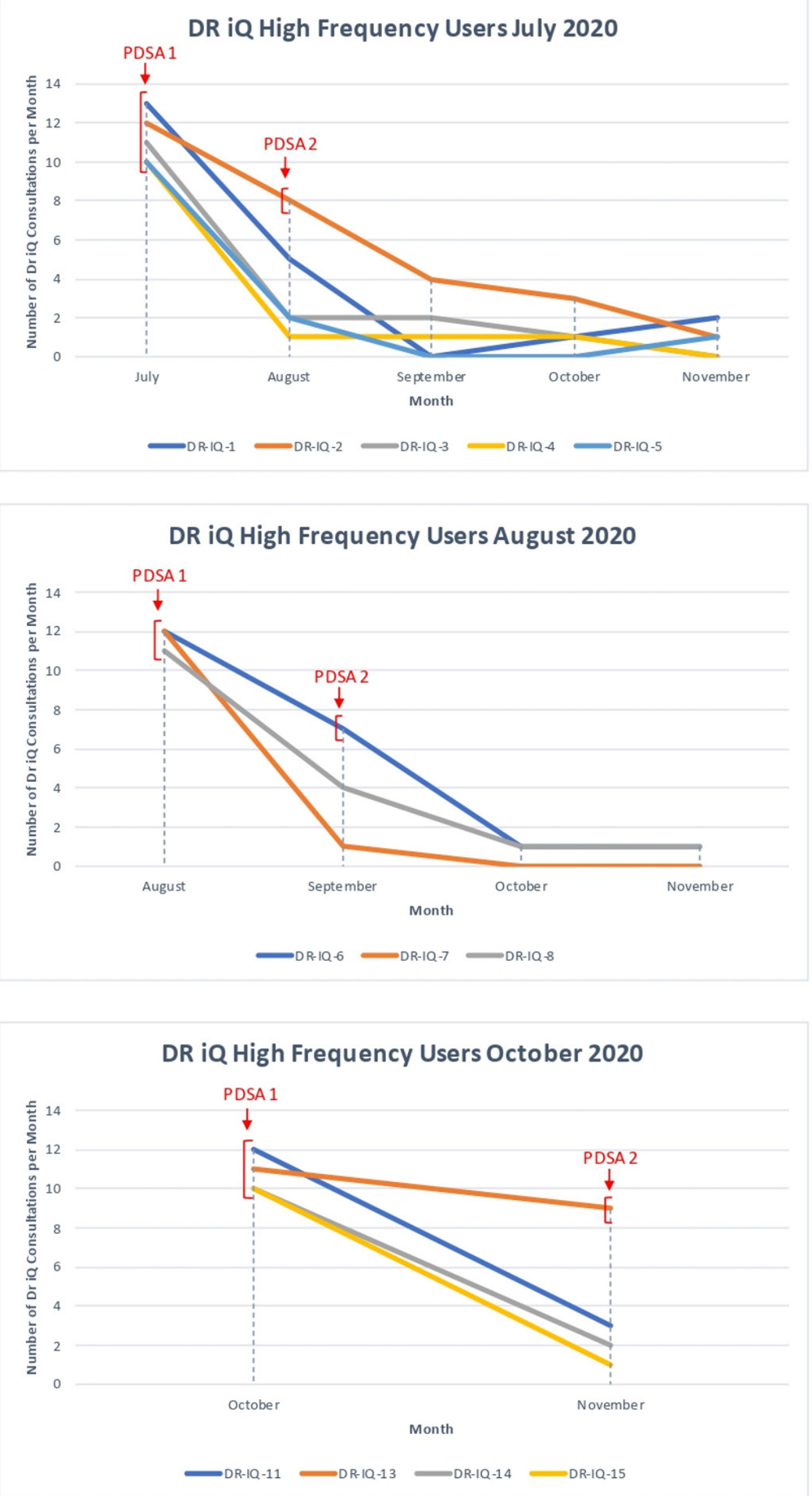

Figure 2 Three graphs illustrating the trend of high frequency users' Dr iQ consultation frequency over time. The point of each PDSA cycle intervention is also signified. Note that users with appropriate high frequency use were not included in the graphs (DR-IQ-9, DR-IQ-10, DR-IQ-12), hence there is no graph for September. PDSA, Plan-Do-Study-Act. 
high frequency users interact with $\mathrm{Dr} \mathrm{iQ}$ and informed us on our change process to ensure unmet health needs were being addressed. It also provided other feedback for the platform, such as design issues which could have potentially led to increased use. For example, one user did not realise that a clinician had responded to their consultation as they did not receive a notification on their device, leading to subsequent submission of frequent consultations. These points were fed back to the platform developers to enhance the patient experience of DriQ.

We presented the project to clinicians in other practices using Dr iQ and it was well received. They felt the interventions could be easily replicated and trialled in different surgeries. Clinicians mentioned although e-consultations may be favoured in terms of increased efficiency, this may not be the case for a subgroup of high frequency users. The intervention of converting high frequency users from using $\mathrm{Dr} i \mathrm{Q}$ to regular appointments may result in an added value of saved time, as we go from $10+$ e-consultations a month taking an estimated 1 hour of time to 1 or 2 telephone or face-to-face appointments taking 10-20 minutes. A number of patients in this group even discharged themselves from regular follow-up after they felt their health needs were met.

The main limitation of this project is that it was focused on one general practice with a small sample size of 12 patients and a relatively young practice population. The average age of our sample was 32.8 years. Our next step would be to apply this intervention on other practices using Dr iQ to assess the effect of the intervention on a larger scale and in different population groups. Another limitation is the lack of data on patient satisfaction and acceptability of our intervention. This could be implemented in a future study through a simple Likert scale questionnaire to assess patient satisfaction before and after the intervention. Additionally, we could have recorded our telephone interviews for transcription and qualitative analysis through thematic analysis. Unfortunately, the technology and expertise was not available in this instance. Future qualitative research specifically studying drivers as well as barriers for e-consultation high frequency use would be useful to inform ideas for effective interventions.

It is difficult to attribute the decrease in consult frequency to the effect of our intervention or to other factors. Cohort studies within a traditional face-toface general practice setting have shown regression in attendance with only one out of every seven patients remaining frequent attenders after 3 years. ${ }^{20}$ Whether this is the case with e-consultations is unknown. As remote consulting technology continues to develop, further research into the pattern of e-consultation use over time as well as the effect of intervention and no intervention between two high frequency user groups would be beneficial. Could high frequency use be naturally limited to the first months after registration as the user is becoming familiar with the platform? Another potential topic of research is to study the overlap between traditional GP HFA and e-consultation high frequency users-are these the same patients and are they shifting from one medium to another? Could the introduction of e-consultations add high frequency users to an existing caseload of HFA?

\section{CONCLUSION}

Online consultations can be beneficial in terms of ease of access, efficiency, and improved patient satisfaction. However, as with a traditional general practice setting, it is likely that high frequency users will arise, and possibly at a higher number due to the ease of access of modern technologies. The reasons for high frequency use are multifactorial. The e-consultation platform may not meet the health needs for a certain subgroup of patients, thereby leading to high frequency usage. These patients often have complex medical backgrounds with underlying chronic physical and mental health problems.

Our project findings suggest that prompt intervention of high frequency users through a multistep approach involving an initial telephone interview assessing their health needs, followed by case discussion in a multidisciplinary team setting and subsequent regular telephone or face-to-face consultations with a named clinician is effective in decreasing high frequency use. Through regular contact with a named clinician, we were able to contain persistent high frequency users to a fortnightly or monthly frequency, decrease inappropriate e-consultation usage, and address unmet health needs.

With the rapid uptake of remote consulting methods recently accelerated by COVID-19, e-consultations and their associated high frequency use is a new and exciting topic for further study. More research is needed to understand the epidemiology and aetiology of high frequency use, which will hopefully advise methods of improvement, improve health needs, and ultimately lead to better patient care.

Contributors $\mathrm{KL}$ and $\mathrm{SQ}$ conceptualised the project, developed the methodology and interpreted the data. KL led the PDSA cycles, engaged key stakeholders, collected data and prepared the initial draft of the manuscript. SQ provided supervision throughout and revised the manuscript.

Funding The authors have not declared a specific grant for this research from any funding agency in the public, commercial or not-for-profit sectors.

Competing interests None declared.

Patient and public involvement Patients and/or the public were involved in the design, or conduct, or reporting, or dissemination plans of this research. Refer to the Methods section for further details.

Patient consent for publication Not required.

Provenance and peer review Not commissioned; externally peer reviewed.

Data availability statement All data relevant to the study are included in the article.

Open access This is an open access article distributed in accordance with the Creative Commons Attribution Non Commercial (CC BY-NC 4.0) license, which permits others to distribute, remix, adapt, build upon this work non-commercially, and license their derivative works on different terms, provided the original work is properly cited, appropriate credit is given, any changes made indicated, and the use is non-commercial. See: http://creativecommons.org/licenses/by-nc/4.0/. 
ORCID iD

Katherine Leung http://orcid.org/0000-0003-3469-9852

\section{REFERENCES}

1 Dr. iQ. The fastest way to access our GP services 7 days a week, including evenings [Internet]. Available: https://dr-iq.com/ [Accessed 3 Dec 2020].

2 Luciano JV, Fernández A, Pinto-Meza A, et al. Frequent attendance in primary care: comparison and implications of different definitions. Br J Gen Pract 2010;60:e49-55.

3 PHE. National General Practice Profiles - PHE [Internet]. Available: https://fingertips.phe.org.uk/profile/general-practice/data\#page/12/ gid/2000005/pat/166/par/E38000186/ati/7/are/F84039/iid/354/age/ 164/sex/4/cid/4/tbm/1/page-options/ovw-do-0_eng-vo-0_eng-do-0_ map-ao-4_cin-ci-4 [Accessed 3 Dec 2020].

4 NHS. nhs-long-term-plan-version-1.2.pdf [Internet]. Available: https://www.longtermplan.nhs.uk/wp-content/uploads/2019/08/nhslong-term-plan-version-1.2.pdf [Accessed 18 Oct 2020].

5 NHS England. Digital First Primary Care [Internet]. Available: https:// www.england.nhs.uk/gp/digital-first-primary-care/ [Accessed 3 Dec 2020].

6 Atherton $\mathrm{H}$, Brant $\mathrm{H}$, Ziebland $\mathrm{S}$, et al. Alternatives to the face-toface consultation in general practice: focused ethnographic case study. Br J Gen Pract 2018;68:e293-300.

7 Brant $\mathrm{H}$, Atherton $\mathrm{H}$, Ziebland $\mathrm{S}$, et al. Using alternatives to face-toface consultations: a survey of prevalence and attitudes in general practice. Br J Gen Pract 2016;66:e460-6.

8 NHS Digital. Appointments in General Practice February 2020 [Internet]. Available: https://digital.nhs.uk/data-and-information/ publications/statistical/appointments-in-general-practice/february2020 [Accessed 3 Dec 2020].

9 RCGP. RCGP survey provides snapshot of how GP care is accessed in latest stages of pandemic [Internet]. Available: https://www.rcgp. org.uk/about-us/news/2020/july/rcgp-survey-provides-snapshot- of-how-gp-care-is-accessed-in-latest-stages-of-pandemic.aspx [Accessed 3 Dec 2020].

10 Wamoscher Z. The returning patient. J Coll Gen Pract 1966;11:166-73.

11 Pasgaard AA, Mæhlisen MH, Overgaard C, et al. Social capital and frequent attenders in general practice: a register-based cohort study. BMC Public Health 2018;18:310.

12 Carmassi C, Dell'Oste V, Ceresoli D, et al. Frequent attenders in general medical practice in Italy: a preliminary report on clinical variables related to low functioning. Neuropsychiatr Dis Treat 2018;15:115-25.

13 Jørgensen JT, Andersen JS, Tjønneland A, et al. Determinants of frequent attendance in Danish general practice: a cohort-based cross-sectional study. BMC Fam Pract 2016;17:9.

14 Vedsted $\mathrm{P}$, Olesen $\mathrm{F}$. Social environment and frequent attendance in Danish general practice. Br J Gen Pract 2005;55:510-5.

15 Reid S, Wessely S, Crayford T, et al. Frequent attenders with medically unexplained symptoms: service use and costs in secondary care. Br J Psychiatry 2002;180:248-53.

16 Neal RD, Heywood PL, Morley S, et al. Frequency of patients' consulting in general practice and workload generated by frequent attenders: comparisons between practices. $\mathrm{Br} J$ Gen Pract 1998;48:895-8.

17 Smits FT, Brouwer HJ, van Weert HCP, van WHC, et al. Predictability of persistent frequent attendance: a historic 3-year cohort study. $\mathrm{Br} J$ Gen Pract 2009;59:e44-50.

18 Haroun D, Smits F, van Etten-Jamaludin F, et al. The effects of interventions on quality of life, morbidity and consultation frequency in frequent attenders in primary care: a systematic review. Eur $J$ Gen Pract 2016;22:71-82.

19 Andersson S-O, Lynöe N, Hallgren C-G, et al. Is frequent attendance a persistent characteristic of a patient? repeat studies of attendance pattern at the family practitioner. Scand J Prim Health Care 2004;22:91-4.

20 Smits FTM, Brouwer HJ, ter Riet G, et al. Epidemiology of frequent attenders: a 3-year historic cohort study comparing attendance, morbidity and prescriptions of one-year and persistent frequent attenders. BMC Public Health 2009;9:36. 